\title{
Development of LEED Apparatus Using Nano-Tips Fabricated by Field-Assisted Etching*
}

\author{
Jo Onoda, ${ }^{\dagger}$ Tomomi Kanaoka, Megumi Kumon, and Seigi Mizuno \\ Department of Molecular and Material Sciences, Kyushu University, Kasuga, Fukuoka 816-8580, Japan
}

(Received 26 January 2012; Accepted 11 April 2012; Published 30 June 2012)

\begin{abstract}
We have developed a low-energy electron diffraction (LEED) apparatus using field emission (FE) from scanning tunneling microscopy (STM) tips. Tips fabricated by field-assisted $\mathrm{H}_{2} \mathrm{O}$ etching enabled us to observe LEED patterns of few layer graphene grown on $\mathrm{SiC}(0001)$ reproducibly. The obtained hexagonal patterns showed a bias dependency and those obtained at sample biases of $93 \mathrm{~V}, 56 \mathrm{~V}$, and $28 \mathrm{~V}$ were interpreted as patterns derived from the graphene, the $\mathrm{SiC}(0001)$ substrate, and a $(6 \sqrt{3} \times 6 \sqrt{3}) \mathrm{R} 30^{\circ}$ buffer layer, respectively. The analysis of Ewald sphere construction indicated that the opening angles of FE from the tip could be deduced directly from the LEED patterns, and their experimentally obtained value was $\sim 13^{\circ}$. From the opening angle of FE and the dataset of tip-to-sample distances, the irradiated areas were estimated at $350 \mathrm{~nm} \phi$ when the sample bias was $100 \mathrm{~V}$.
\end{abstract}

[DOI: 10.1380/ejssnt.2012.292]

Keywords: Field ion microscopy; Etching; Field emission; Low-energy electron diffraction (LEED); Tungsten; Nano-tip; Graphene

\section{INTRODUCTION}

The development of new apparatuses based on scanning tunneling microscopy (STM) receives considerable attention because they will provide techniques with high spatial resolution. For example, it was shown that four-tip STM could measure the surface electric conductivity on a sub-micron scale [1, 2]. Field emissions (FEs) from STM tips also have the potential to probe small surface areas with electron beams. Topografiner, which was developed by Young et al. in 1971 before the invention of STM for secondary electron mapping of metallic surfaces, could provide the microtopography with a vertical resolution of $3 \mathrm{~nm}$ and a horizontal resolution of $400 \mathrm{~nm}$ in the plane of the surface [3, 4]. Kirk et al. also attempted secondary electron mapping of surfaces using a near-field emission scanning electron microscope (NFESEM) and estimated the resolution to be $\sim 2 \mathrm{~nm}$ from the sharpness of the step edge $[5,6]$. On the other hand, Fink et al. developed the projection microscope $[7,8]$, which can image carbon fibers using transmitted electrons with high spatial resolution of better than $10 \mathrm{~nm}$. In order to obtain structural information of a single molecule, recently their studies focused on the fabrication of micrometer-sized electron lenses [9], a phase retrieval algorithm, and a large-area high-resolution detector. Reihl et al. demonstrated a scanning Auger microscope using a field-emission tip [10]. Despite the efforts of these studies, FE microscopes based on the scanning probe microscopy (SPM) technique have not yet come into wide use.

Low-energy electron diffraction (LEED) is known as one of the most powerful tools for determining wellordered surface structures. However, its typical probing area is relatively large ( $\sim 0.5 \mathrm{~mm}$ probing diameter $)$. Bauer et al. developed a low-energy electron microscope (LEEM) in which the electron beams can converge to sub-

* This paper was presented at the 6th International Symposium on Surface Science - Towards Nano, Bio and Green Innovation-, Tower Hall Funabori, Tokyo, Japan, December 11-15, 2011.

†Corresponding author: joonoda@kyudai.jp micron size through the use of magnetic lenses [11], and Tromp et al. could obtain LEED patterns from an area as small as $200 \mathrm{~nm}$ [12]. On the other hand, we have attempted to combine the LEED technique with SPM to obtain diffraction patterns from a small region, about $100 \mathrm{~nm} \phi$, without the guidance of magnetic lenses [13-15]. This instrument detects backscattered electrons; therefore, samples are not restricted to thin films as in the case of transmission electron microscopy (TEM). Moreover, it would be possible to obtain STM images and LEED patterns of the same region. So far, we have successfully observed LEED patterns of a $\mathrm{Cu}(001)$ surface, which could be derived from $4 \mu \mathrm{m} \phi$ surface area [16]. Some critical problems, however, still remain with regard to the structure of the tip apex and the resultant properties of $\mathrm{FE}$, whose features need to be controlled in a reproducible way. It is known that the field-assisted gas etching method enables us to sharpen the apex shape in the range from atomic scale to several tens of nanometers [1719]. Especially, a modified sharpening procedure [20, 21] can yield a highly collimated field emitter which could be suitable for our LEED experiments. In this paper, the application of nano-tips to our new LEED apparatus is shown. Hexagonal LEED patterns of graphene grown on an $\mathrm{SiC}(0001)$ substrate were observed by this apparatus. Irradiated areas were also estimated by measuring the opening angles of incident beams and the tip-to-sample distances.

\section{EXPERIMENTAL}

The apparatus mainly consists of a preparation chamber and a main chamber. In the preparation chamber $\left(10^{-8} \mathrm{~Pa}\right)$, tips were treated by field ion microscopy (FIM) and field emission microscopy (FEM) (35 $\mathrm{mm} \phi \mathrm{MCP})$. Polycrystalline $\mathrm{W}\langle 110\rangle$ oriented wires $(0.2 \mathrm{~mm} \phi)$ that were electrochemically polished in $2-\mathrm{M} \mathrm{NaOH}$ solution were fabricated by field-assisted $\mathrm{H}_{2} \mathrm{O}$ etching at room temperature as described in Ref. [20] to obtain nano-tips with high collimation ability. Few-layer graphene grown on $\mathrm{SiC}(0001)$ [22] was prepared by annealing and characterized by equipped conventional LEED in the prepara- 


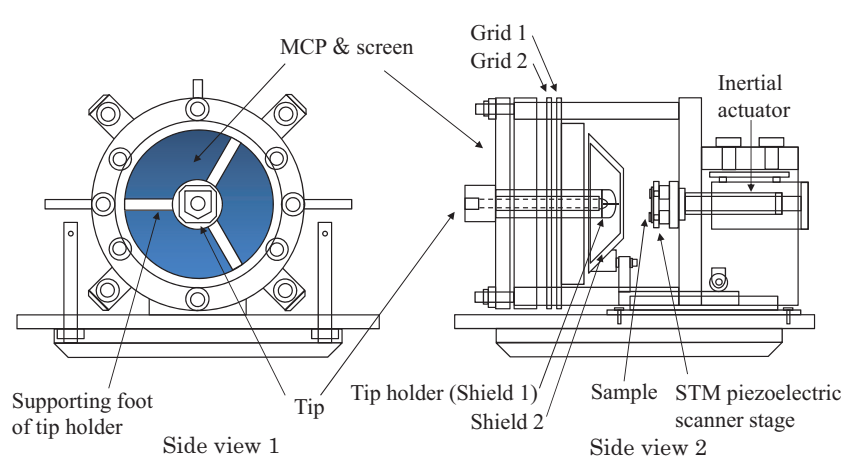

FIG. 1: Schematic illustrations of the FE-LEED apparatus.

tion chamber. An etched tip and a sample can be transferred from the preparation chamber to the main chamber $\left(10^{-8} \mathrm{~Pa}\right)$, respectively. Figure 1 represents the side views of our new field emission-type LEED (FE-LEED) system. The tip fabricated in the preparation chamber beforehand was inserted into the tip holder equipped in the center hole of another MCP (42 $\mathrm{mm} \phi)$ and grounded. The sample was subjected to positive bias voltage and its position was adjusted by an actuator and a piezoelectric scanner to obtain a suitable emission current (typically $0.2 \mathrm{nA}$ ). The electrons emitted from the tip apex approached the sample surface at the normal direction. Backscattered electrons were intensified by MCP and displayed by a phosphor screen, and then LEED patterns were captured by a CCD camera located behind the phosphor screen. The tip holder was insulated from the tip and acted as shield 1. Shield 2 could be coarsely moved toward the normal direction by the inertial actuator. Positive bias voltages could be applied to shields 1 and 2 independently, which were adjusted to realize proper electron trajectories for the FE-LEED patterns. Grids 1 and 2 were located in front of the MCP. Positive bias voltages could also be applied to grids 1 and 2; the former was set for the purpose of drawing all scattered electrons and the latter was for the removal of inelastically scattered electrons. For achieving symmetrical electrostatic potential around the tip apex, the tip holder (shield 1) was designed to be a cylinder with a hemispheric cap while shield 2 had a round shape. Three supporting feet of tip holder were behind the screen; thus, their shadows appeared during the observation of FE-LEED patterns.

\section{RESULTS AND DISCUSSIONS}

\section{A. Nano-tip fabricated by field-assisted $\mathrm{H}_{2} \mathrm{O}$ etching}

Figure 2(a) represents the FIM image of the tip whose apex was cleaned by field evaporation in He at a pressure of $1 \times 10^{-3} \mathrm{~Pa}$. Subsequently, $\mathrm{H}_{2} \mathrm{O}$ was additionally introduced up to $1.3 \times 10^{-3} \mathrm{~Pa}$, and then field-assisted $\mathrm{H}_{2} \mathrm{O}$ etching was performed at the fixed bias of $4.0 \mathrm{kV}$ [20]. As the continuous field evaporation and the $\mathrm{H}_{2} \mathrm{O}$ etching progressed, a ring-like $\mathrm{H}_{2} \mathrm{O}$ imaging region gradually appeared from the periphery of the tip as shown in Fig. 2(b). At this stage, it was hard to clearly observe the tip apex
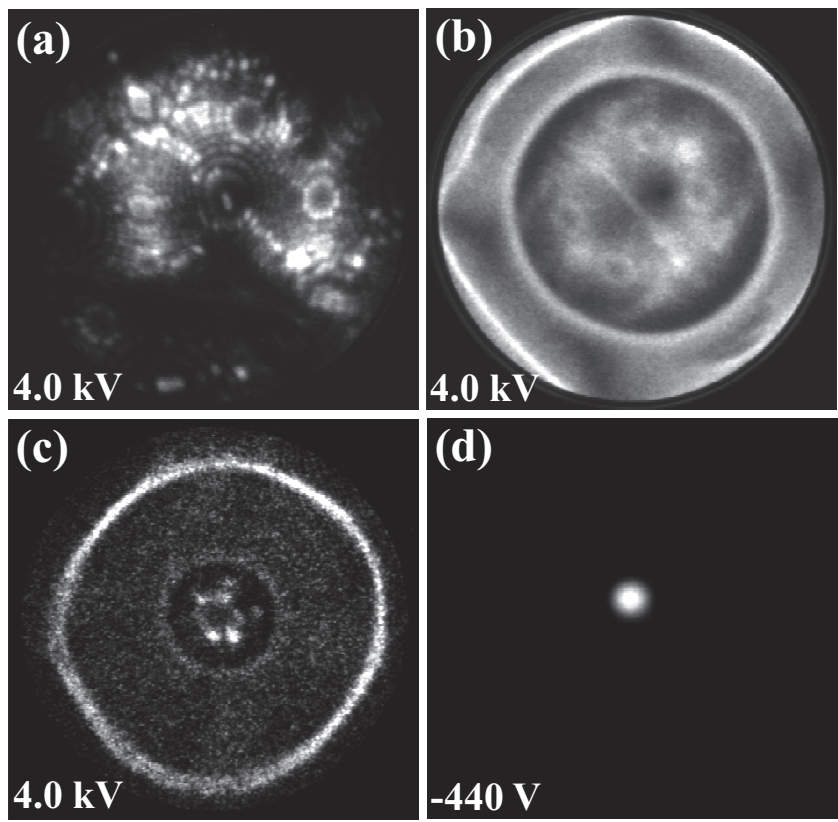

FIG. 2: Fabrication of nano-tip. (a)-(c) FIM images during field-assisted $\mathrm{H}_{2} \mathrm{O}$ etching. The bias voltage was fixed at $4.0 \mathrm{kV}$. (d) FEM image after stabilizing the tip apex by the FDG method.

structure by He imaging, but we could still recognize the four equivalent (112) planes surrounding the centered (110) plane. When a nano-protrusion, which solely revealed the (110) plane, was formed on the large base of the tungsten tip as represented in Fig. 2(c), we stopped the introduction of $\mathrm{H}_{2} \mathrm{O}$. After the apex modification by field-assisted $\mathrm{H}_{2} \mathrm{O}$ etching, we switched to FEM mode and stabilized the tip apex structure by the field-enhanced diffusion growth (FDG) method [23]. Figure 2(d) represents the FEM image at the bias voltage $-440 \mathrm{~V}$, with an emission current of $150 \mathrm{pA}$. Its opening angle of $\mathrm{FE}$ defined by full width at half maximum (FWHM) became $5.3^{\circ}$.

\section{B. FE-LEED observation}

Normally, we mounted $\mathrm{H}_{2} \mathrm{O}$ etched tips which showed an opening angle of $5^{\circ}$. As a result, we could reproducibly observe the FE-LEED patterns of few-layer graphene on $\mathrm{SiC}(0001)$ as shown in Figs. 3(a)-(c). In the cases that the tips had several emission sites on their apexes or large opening angles of FE, we hardly measured the stable patterns and the datasets of the tip-to-sample distance.

\section{Interpretation of FE-LEED patterns of graphene on $\operatorname{SiC}(0001)$}

Figure 4 shows the potential energy diagram of electrons in the FE-LEED apparatus. For ease of understanding, the left part from the tip to the sample depicts the incident beam while the right part from the sample to the MCP depicts the backscattered beam. $E_{\mathrm{V}}, E_{\mathrm{F}}$ and $E_{0}$ represent the vacuum level, the Fermi level, and the inner potential, and $\phi_{\mathrm{T}}$ and $\phi_{\mathrm{S}}$ are work functions of the 

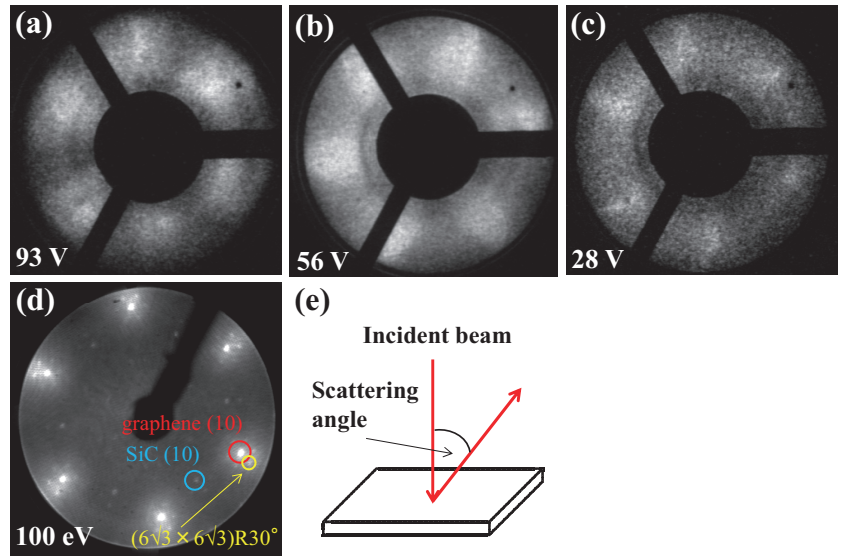

(e)

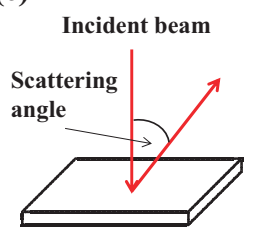

FIG. 3: (a)-(c) FE-LEED patterns obtained and (d) a conventional LEED pattern of graphene on $\mathrm{SiC}(0001)$. (10) spots from the graphene and $\mathrm{SiC}(0001)$ substrate are indicated by red and blue circles while a spot derived from the $(6 \sqrt{3} \times 6 \sqrt{3}) \mathrm{R} 30^{\circ}$ buffer layer is represented by yellow circle. (e) The definition of the scattering angle.

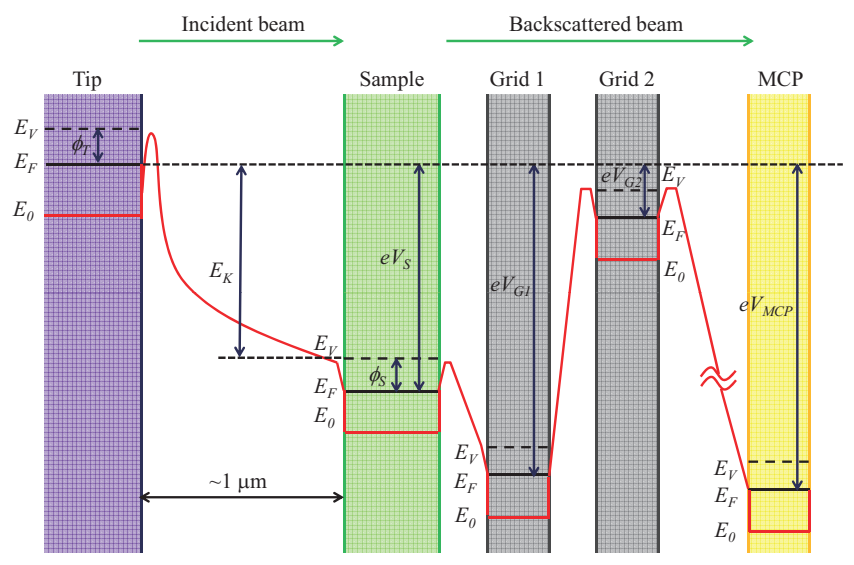

FIG. 4: Potential energy diagram of electrons in the FE-LEED apparatus.

tip and sample, respectively. The tip is grounded while the sample, grid 1, grid 2, and the input of MCP are the applied bias voltage $V_{\mathrm{S}}, V_{\mathrm{G} 1}, V_{\mathrm{G} 2}$, and $V_{\mathrm{MCP}}$, respectively. The kinetic energy of electrons in front of the sample, defined as $E_{\mathrm{K}}$ in Fig. 4, becomes

$$
E_{\mathrm{K}}=e V_{\mathrm{S}}-\phi_{\mathrm{S}} \quad(\mathrm{eV})
$$

where $e$ is the electron charge. Figure 3(d) represents the conventional LEED pattern of graphene on $\mathrm{SiC}(0001)$ at $100 \mathrm{eV}$, which is characterized by the brightest hexagonal (10) spots derived from graphene as indicated by the red circle. Each spot is also surrounded by six spots originating from the interface layer between the graphene and SiC substrate, known as the $(6 \sqrt{3} \times 6 \sqrt{3})$ R30 $0^{\circ}$ buffer layer (yellow circle). The hexagonal (10) spots derived from the $\mathrm{SiC}(0001)$ substrate appear at the inside of the graphene LEED pattern and rotated by $30^{\circ}$ as represented by the blue circle. Figures 3(a)-(c) shows FE-LEED patterns of the same sample obtained at sample biases of $93 \mathrm{~V}$, $56 \mathrm{~V}$, and $28 \mathrm{~V}$, respectively. The hexagonal patterns were visible and their orientations of spots were the same (a)

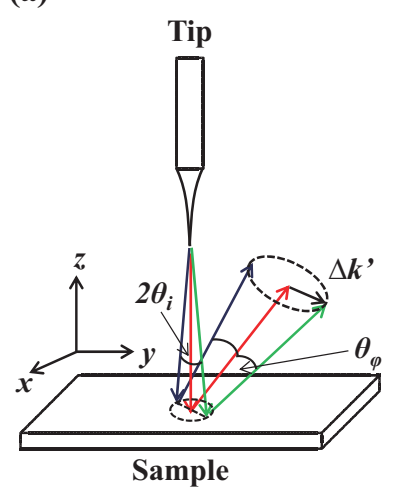

(c)

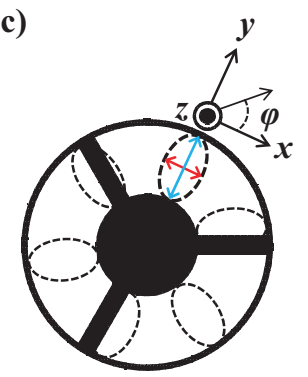

(b)

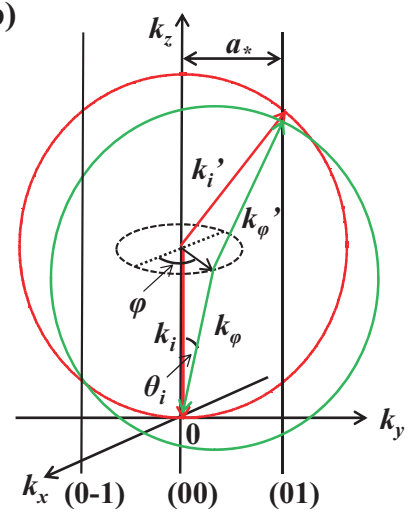

(d)

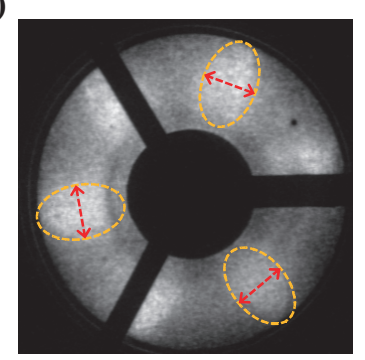

FIG. 5: (a) Schematic illustration of the incident and diffracted electron beams with opening angle $2 \theta_{\mathrm{i}}$ in real space. The opening angle of the scattered electron beam is $\theta_{\varphi}$. (b) The Ewald constructions of normal- and tilting-incidence electron beams. (c) The orientation correspondence between the spot and coordinates. (d) The denotation of FWHM of spots.

as those of the conventional LEED pattern of graphene except for the one at $56 \mathrm{~V}$. Here, we could consider that either of the FE-LEED patterns at $93 \mathrm{~V}$ or $28 \mathrm{~V}$ was derived from graphene (10) spots. However, it was calculated that their scattering angles (defined in Fig. 3(e)) were $37^{\circ}$ and $90^{\circ}$, respectively; thus, it was more reasonable that only the spots of the FE-LEED pattern at $93 \mathrm{~V}$ could be graphene (10). If so, those obtained at $56 \mathrm{~V}$ were supposed to be (10) spots derived from the $\mathrm{SiC}(0001)$ substrate. Actually, this presumption was supported by the result that the scattering angle of (10) spot of $\mathrm{SiC}(0001)$ was calculated to be $38^{\circ}$ at $56 \mathrm{~V}$. Therefore, the spots in the FE-LEED pattern at $28 \mathrm{~V}$ were considered to be derived from the $(6 \sqrt{3} \times 6 \sqrt{3}) \mathrm{R} 30^{\circ}$ buffer layer.

It could be also considered that these rotations originated from the effects of stray electric/magnetic fields. However, since shields 1 and 2 have cylindrical symmetry, the electric field does not affect the trajectories of scattered electrons to rotate their orientation. Moreover, since the apparatus was so designed as to avoid magnetic disturbance by using high-permeability material, scattered electrons also cannot be deflected by stray magnetic fields. Actually, the changes from (a) to (b) and from (b) to (c) in Fig. 3 did not strictly displace one another, such that as one hexagonal pattern appeared with the decrease of $V_{\mathrm{S}}$, another disappeared; rather, we could observe the coexistence of two different patterns at sample biases of around $70 \mathrm{~V}$ and $40 \mathrm{~V}$. There is also another cause to be considered: inelastically scattered electrons from the sample. They might have been detected at MCP and formed hexagonal secondary electron patterns as mentioned in 
Ref. [24]. We tried to decrease or increase inelastic electrons by adjusting $V_{\mathrm{G} 2}$ but the LEED patterns did not change drastically and only got clearer or blurrier. Therefore, the FE-LEED patterns appear to preserve the symmetry of the patterns of elastically scattered electrons and must not be influenced by those of the inelastic electrons.

\section{Evaluation of the opening angle of FE and estimation of} the irradiated area

We can calculate the diameter of the irradiated area $S\left(V_{\mathrm{S}}\right)$ at sample bias $V_{\mathrm{S}}$ by the following equation.

$$
S\left(V_{\mathrm{S}}\right)=2 d\left(V_{\mathrm{S}}\right) \tan \theta_{\mathrm{i}}
$$

Here, $d\left(V_{\mathrm{S}}\right)$ is the tip-to-sample distance at sample bias $V_{\mathrm{S}}$; this is because the FE-LEED was operated under constant current mode. The angle $\theta_{\mathrm{i}}$ is the semi-opening angle of the incident electron beam and could be obtained directly from the FE-LEED patterns as described in the following discussion. Figure 5(a) shows the schematic il- lustration of the incident electron beams with the opening angle $2 \theta_{\mathrm{i}}$ in real space. It is supposed that the incident beams normal to the sample surface (indicated as red arrow) have the maximum FE intensity distribution and the tilting incident beams (indicated as green and blue arrows) have half of the maximum. The scattered electron beams can be obtained by utilizing Ewald constructions as defined in Fig. 5(b) and should form the spots whose opening angle becomes $\theta_{\varphi}+\theta_{\varphi+180^{\circ}}$. Here, $\varphi$ is considered to range from 0 to 180 degrees. Since $\boldsymbol{k}_{\mathbf{i}}^{\prime}$ and $\boldsymbol{k}_{\boldsymbol{\varphi}}^{\prime}$ are expressed as

$$
\begin{aligned}
\boldsymbol{k}_{\mathrm{i}}^{\prime}= & \left(\begin{array}{c}
0 \\
a^{*} \\
\sqrt{k_{\mathrm{i}}^{\prime 2}-a^{* 2}}
\end{array}\right) \\
\boldsymbol{k}_{\boldsymbol{\varphi}}^{\prime}= & \left(\begin{array}{c}
-k_{\mathrm{i}} \sin \theta_{\mathrm{i}} \cos \varphi \\
a^{*}-k_{\mathrm{i}} \sin \theta_{\mathrm{i}} \sin \varphi \\
\sqrt{k_{\mathrm{i}}^{2}\left(1-\sin ^{2} \theta_{\mathrm{i}}\right)+2 a^{*} k_{\mathrm{i}} \sin \theta_{\mathrm{i}} \sin \varphi-a^{* 2}}
\end{array}\right)
\end{aligned}
$$

$\theta_{\varphi}$ is described as

$$
\theta_{\varphi}=\cos ^{-1}\left(\frac{a^{*}}{k_{\mathrm{i}}}\right)^{2}-\frac{a^{*}}{k_{\mathrm{i}}} \sin \theta_{\mathrm{i}} \sin \varphi+\sqrt{1-\left(\frac{a^{*}}{k_{\mathrm{i}}}\right)^{2}} \sqrt{\cos ^{2} \theta_{\mathrm{i}}+2\left(\frac{a^{*}}{k_{\mathrm{i}}}\right) \sin \theta_{\mathrm{i}} \sin \varphi-\left(\frac{a^{*}}{k_{\mathrm{i}}}\right)^{2}} .
$$

The spot shape of FE-LEED could be expressed by

$$
\Delta \boldsymbol{k}^{\prime}=\boldsymbol{k}_{\varphi}^{\prime}-\boldsymbol{k}_{\mathbf{i}}^{\prime}
$$

which is represented in Fig. 5 (a).

Figure 5(c) represents the orientation correspondence between one of the spots in the FE-LEED pattern and the coordinates indicated in Fig. 5 (a). Actually, as already mentioned, shield 1 and 2 have cylindrical symmetry; therefore, the electric field between the shields does not affect the opening angle of the scattered electron beams to the azimuth direction as represented by the red arrow in Fig. 5(c) (corresponding to the case of $\phi=0^{\circ}$ ), while the electric fields of shields could influence the opening angle to the radial direction as shown by the blue arrow in Fig. 5(c) (corresponding to the case of $\phi=90^{\circ}$ ); therefore, the most important case is $\phi=0^{\circ}$. We calculated $\theta_{0^{\circ}}+\theta_{180^{\circ}}$ using Eq. (5) for graphene $\left(a^{*}=2.95 \AA^{-1}\right)$ and the $\operatorname{SiC}(0001)\left(a^{*}=2.36 \AA^{-1}\right)$ substrate in the range such that $2 \theta_{\mathrm{i}}$ is 2 to 18 degrees and $E_{\mathrm{k}}$ is 25 to $100 \mathrm{eV}$. However, all the results showed that $\theta_{0^{\circ}}+\theta_{180^{\circ}}$ became similar to $2 \theta_{\mathrm{i}}$. Therefore, the above considerations should support that we can almost regard the opening angle of the scattered electron beams in FE-LEED, experimentally estimated as the red arrow in Fig. 5(d), as those of incident electron beams. The averaged opening angle of the incident electron beam in the range of $V_{\mathrm{S}}$ from 30 to $100 \mathrm{~V}$ became $\sim 13^{\circ}$, representing an almost $2-3$ times larger value than that in FEM; $\sim 5^{\circ}$. This could represent that the field distribution in the proximity of the tip apex did not cause enough suppression of the electron beam toward the longitudinal direction because of the close tipto-sample distance of $\sim 1 \mu \mathrm{m}$.

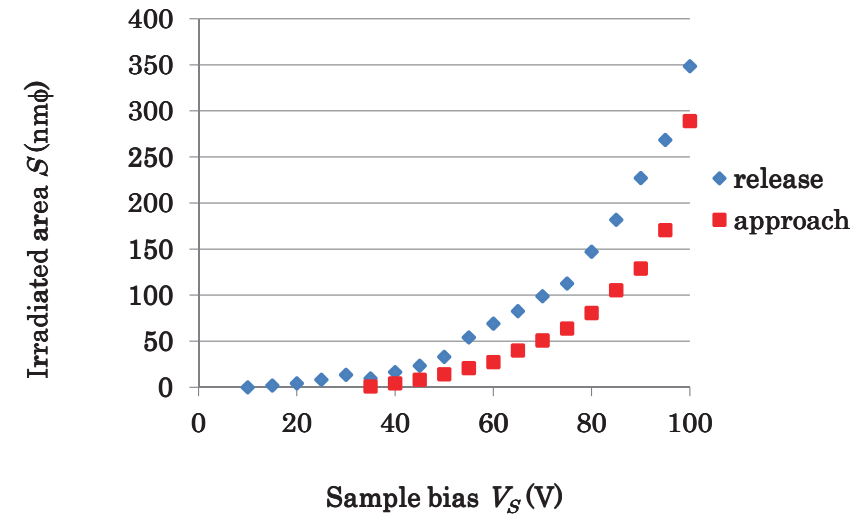

FIG. 6: Estimation of irradiated area under the constant current mode $(0.25 \mathrm{nA})$.

From the typical datasets of the tip-to-sample distance, which were measured while the tip was releasing and approaching under a constant current, $0.25 \mathrm{nA}$, we calculated the irradiated area using Eq. (2) and the results are represented in Fig. 6. This should mean that we could succeed in obtaining the LEED patterns from the surface area (Figs. 3(a)-(c)) less than $350 \mathrm{~nm} \phi$. Generally, the fact that the irradiated area is relatively small can cause the broadening of beam spots in FE-LEED patterns due to the Laue function. Thus, the careful consideration will be needed when we evaluate the opening angle of scattered electron beam, especially at the lower bias region where the close tip-to-sample distance and the small illuminated 
area are simultaneously realized.

In the current experiments, we only applied the sample bias up to $100 \mathrm{~V}$ due to the limitation of bias voltage in the FE-LEED unit. If we could have increased the bias up to $200-300 \mathrm{~V}$, it is expected that the reduction of the opening angle would compensate for the enlargement of the irradiated area, caused by the tip displacement from the sample. In addition, because we used fewlayer graphene which was uniformly grown on $\mathrm{SiC}(0001)$, FE-LEED patterns peculiar to the small surface region had not been observed. For our future work, we will attempt to probe samples such as $\mathrm{Si}(100)$ or highly oriented pyrolytic graphite (HOPG), which both contain domains of $\sim 100 \mathrm{~nm} \phi$.

\section{CONCLUSIONS}

Tips fabricated by field-assisted $\mathrm{H}_{2} \mathrm{O}$ etching, which typically had an opening angle of $\sim 5^{\circ}$, enabled us to observe FE-LEED patterns of graphene grown on $\mathrm{SiC}(0001)$ reproducibly. The obtained patterns showed a bias dependency; the orientation of spots at $93 \mathrm{~V}$ and $28 \mathrm{~V}$ of sample biases were coincident with those of conventional LEED patterns while those at $56 \mathrm{~V}$ were rotated by $30^{\circ}$. Taking the scattering angle into consideration, it seemed that the patterns obtained at $93 \mathrm{~V}, 56 \mathrm{~V}$, and $28 \mathrm{~V}$ were formed by the elastically scattered electrons derived from the graphene layers, the $\mathrm{SiC}(0001)$ substrate, and the $(6 \sqrt{3} \times 6 \sqrt{3}) \mathrm{R} 30^{\circ}$ buffer layer, respectively. On the other hand, for evaluating the irradiated area, the opening angle of FE and the dataset of tip-to-sample distance are necessary. From the analysis of Ewald sphere construction, it was found that the opening angles of FE could be obtained directly from the FE-LEED patterns. The average value of the opening angles deduced from experimentally obtained spots was $\sim 13^{\circ}$, which was $2-3$ times larger than that in FEM: $\sim 5^{\circ}$. The reason should be the close tip-to-sample distance, which could have caused the diminution of the suppression effect of the large base on diverged electron beams. Irradiated areas during FELEED experiments were estimated at less than $350 \mathrm{~nm} \phi$.
[1] I. Shiraki, F. Tanabe, R. Hobara, T. Nagao, and S. Hasegawa, Surf. Sci. 493, 4256 (2001).

[2] S. Hasegawa, I. Shiraki, F. Tanabe, and R. Hobara, Curr. Appl. Phys. 2, 633 (2001).

[3] R. Young, J. Word, and F. Scire, Phys. Rev. Lett. 27, 922 (1971).

[4] R. Young, J. Word, and F. Scire, Rev. Sci. Instrum. 43, 999 (1972).

[5] T. L. Kirk, L. G. De Pietro, D. Pescia, and U. Ramsperger, Ultramicroscopy 109, 463 (2009).

[6] T. L. Kirk, O. Scholder, L. G. De Pietro, U. Ramsperger, and D. Pescia, Appl. Phys. Lett. 94, 153502 (2009).

[7] M. Germann, T. Latychevskaia, C. Escher, and H.-W. Fink, Phys. Rev. Lett. 104, 095501 (2010).

[8] E. Steinwand, J.-N. Longchamp, and H.-W. Fink, Ultramicroscopy 111, 282 (2011).

[9] T. Latychevskaia and H.-W. Fink, Phys. Rev. Lett. 98, 233901 (2007).

[10] B. Reihl and J. K. Gimzewski, Surf. Sci. 189/190, 36 (1987).

[11] E. Bauer, Ultramicroscopy 17, 51 (1985).

[12] R. M. Tromp, M. Mankos, M. C. Reuter, A. W. Ellis, and M. Copel, Surf. Rev. Lett. 5, 1189 (1998).

[13] S. Mizuno, J. Vac. Sci. Technol. B 19, 1874 (2001).
[14] S. Mizuno. J. Fukuda, and H. Tochihara, Surf. Sci. 514, 291 (2002).

[15] S. Mizuno, J. Fukuda, M. Iwanaga, and H. Tochihara, Jpn. J. Appl. Phys. 43, 5501 (2004).

[16] S. Mizuno, F. Rahman, and M. Iwanaga, Jpn. J. Appl. Phys. 45, L178 (2006).

[17] M. Rezeq, J. Pitters, and R. Wolkow, J. Chem. Phys. 124, 204716 (2006).

[18] J. Onoda, F. Rahman, and S. Mizuno, e-J. Surf. Sci. Nanotechnol. 6, 152 (2008).

[19] J. Onoda, S. Mizuno, and H. Ago, Surf. Sci. 604, 1094 (2010).

[20] J. Onoda, S. Mizuno, Appl. Surf. Sci. 257, 8427 (2011).

[21] Y. Sugiura, H. Liu, T. Iwata, S. Nagai, K. Kajiwara, K. Asaka, Y. Saito, and K. Hata, e-J. Surf. Sci. Nanotechnol. 9, 344 (2011).

[22] U. Starke and C Riedl, J. Phys.: Condens. Matter 21, 134016 (2009).

[23] K. Nagaoka, H. Fujii, K. Matsuda, M. Komori, Y. Murata, C. Oshima, and T. Sakurai, Appl. Surf. Sci. 182, 12 (2001).

[24] H. Hibino, H. Kageshima, F.-Z. Guo, F. Maeda, M. Kotsuzi, and Y. Watanabe, Appl. Surf. Sci. 254, 7596 (2008). 